\title{
Diagnostics of warm dense matter by high-resolution X-ray spectroscopy of hollow ions
}

\author{
A. YA. FAENOV,$^{1,2}$ I. YU. SKOBELEV, ${ }^{2,3}$ T. A. PIKUZ, ${ }^{1,2}$ S. A. PIKUZ, JR., ${ }^{2,3}$ R. KODAMA, ${ }^{1}$ AND \\ V. E. FORTOV ${ }^{2}$ \\ ${ }^{1}$ Institute for Academic Initiatives, Osaka University, Suita, Osaka, Japan \\ ${ }^{2}$ Joint Institute for High Temperatures, Russian Academy of Sciences, Moscow, Russia \\ ${ }^{3}$ National Research Nuclear University MEPhI, Moscow, Russia \\ (ReceIved 5 September 2014; ACCEPTEd 26 October 2014)
}

\begin{abstract}
The short review on the possible applications of the hollow ion spectra for the diagnostics of the high-temperature plasma created by intensive laser and particle beams is presented. Because of the hollow ion spectra features are defined mainly by the mechanisms of their excitation, we consider the various types of the high-temperature plasma where different excitation processes are important. It is shown that like ordinary spectral lines, spectra of the hollow ions offer considerable diagnostic opportunities. At the present time, hollow ion spectra are used mainly to investigate plasma heated by X-ray radiation, but the hollow ions must be generated when plasma is heated by fast heavy ion beams too. In this case, the resultant substance state will be also characterized by solid-state density, and some spatial regions of targets will have relatively low temperatures, i.e., will be a nonideal plasma. It is emphasized that hollow ion spectra are promising diagnostic tool for both nonideal plasma and warm dense matter.
\end{abstract}

Keywords: Hollow ions; Laser plasma diagnostics; Ultra-intense ultra-short laser interaction with matter; Warm dense matter; X-ray spectroscopy

\section{INTRODUCTION}

The X-ray emissions from plasma produced by low contrast optical or infrared laser pulses of picosecond and femtosecond duration, begun being investigated in details during the past 20 years. It was found that the main spectral features of this radiation is rather similar to the previously observed in experiments with a long (nanosecond) laser pulses. The main features of such spectra are strong resonance line emission from closed-shell ions surrounding well-resolved redside satellite emission. With increasing intensity of laser pulses $\left(>10^{17} \mathrm{~W} / \mathrm{cm}^{2}\right)$, cold characteristic line started to be generated by non-thermal electrons. In contrast, experiments performed with a high-contrast (about $10^{10}$ ) femtosecond lasers (Faenov et al., 1997; Urnov et al., 1998) allowed to observe for the first time a new type of spectra. The observed "unusual" spectra differed from the more usual ones by presence of complex spectral structures of the quasicontinuous nature. For such type of spectra, the main

Address correspondence and reprint requests to: A. Ya. Faenov, Joint Institute for High Temperatures, Russian Academy of Sciences, Izhorskaya 13 bld.2, Moscow, 125412 Russia. E-mail: anatolyf@hotmail.com resonance lines have low intensities and are practically unresolved. This new type of spectra (Faenov et al., 1997; 1999; Urnov et al., 1998; Maksimchuk et al., 2000) is caused by radiation transitions in the multi-charged $\mathrm{K}^{2}$-hollow ions, i.e., ions with a completely empty K-shell (see Fig. 1; for more details, see Skobelev et al., 2012). Transitions of this kind were also observed in experiments on the interaction of the ion beams (Briand et al., 1996; 1990; 1997; Bailey et al., 1990; Limburg et al., 1995; Ninomiya et al., 1997; Winter et al., 1999; Schenkel et al., 1999; McMahon et al., 2011; Rzadkiewicz et al., 2010), synchrotron radiation (Diamant et al., 2000b; 2000a; 2001; 2003), or X-ray free electron laser beams (Vinko et al., 2012) with a solid target. The first observation of the similar emission pattern for multi-charged ions was done at the Naval Research Laboratory's NIKE KrF laser facility using ns laser pulses (Aglitsky et al., 1996).

The spectra of the new type, i.e., spectra of the hollow ions, as well as conventional spectral lines have high diagnostic capabilities. A very important motivation for the development of theoretical and experimental hollow ion spectroscopy is the fact that these spectra should be excited 


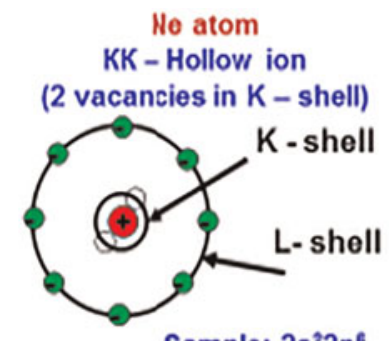

Sample: $2 s^{2} 2 p^{6}$

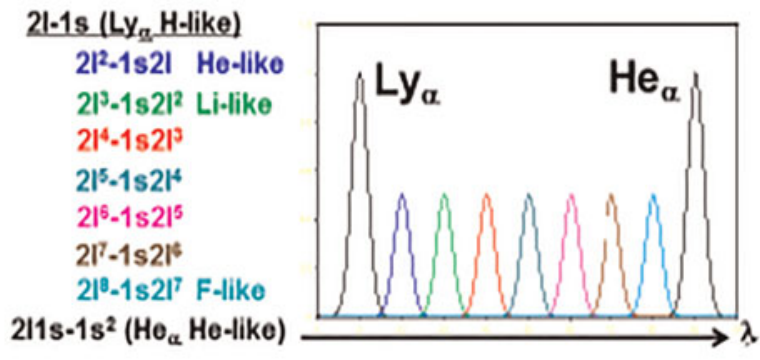

$\mathrm{KL}$ - type of Hollow ions

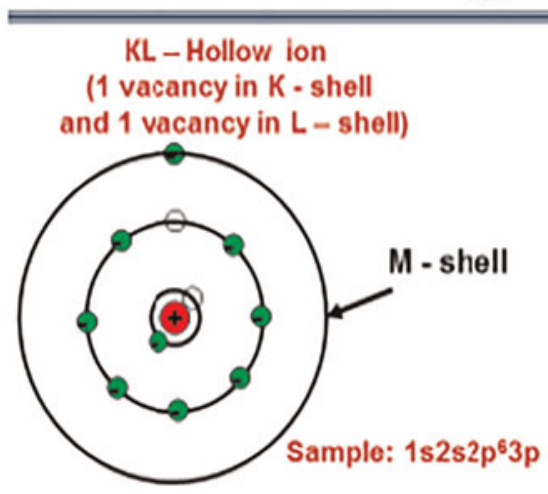

$1 \mathrm{~s} 2 \mathrm{p}-1 \mathrm{~s}^{2}\left(\mathrm{Ho}_{\mathrm{a}} \mathrm{Ho}\right.$-like)

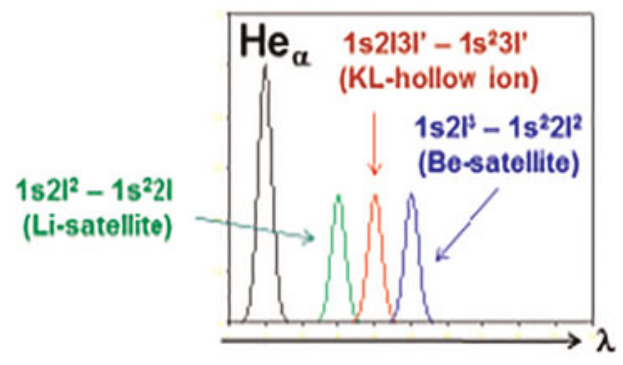

Fig. 1. KK and KL Hollow ions structure and spectral ranges where they could be observed.

in warm dense matter and non-ideal plasma. Since these spectra are caused by the transitions of electrons between deep inner ion shells, the coupling effects must have only little influence on the spectra of hollow ions. It means that the hollow ion spectra can be used for X-ray spectra diagnostic of non-ideal plasma without taking into account nonideality effects in spectra calculations. Additional advantages of using hollow ion emission for diagnostics of hot dense plasma is lower optical depth compare with the large optical depths of conventional emission lines, which limit diagnostic utility of the usual spectral lines.

A significant amount of hollow ions in plasma could be reached if a mechanism that efficiently removes electrons mainly from the inner ion shells exist. It has been shown recently that such a mechanism does exist, and there are some processes in which the probability of removing electrons from the inner-shells is at least not too small compared with the outer-shell ionization. Generally speaking, the ionization of ions in plasma can occur upon collision of an ion with an electron, ion, or photon.

In ion-ion collisions, both non-resonant ionization by the Coulomb field of the incident ion and the resonant charge-exchange are possible. The ion impact ionization cross-sections are large only for ultra-high energy ions, which are at present efficiently produced only in ion accelerators (Fortov, 2009). It is necessary to mention that the first observations of the spectra of hollow atoms were done in accelerator experiments (Briand et al., 1990). At the same time for laser-produced plasma such mechanism is not very important, because there are serious difficulties to produce ultra-high energy ions in this case. Resonant charge exchange is another mechanism that can produce different states of hollow ions if the interaction of ions with significantly different multiplicities occurs. Typical example of such process was demonstrated when laser-produced plasma expands into a residual gas (Rosmej et al., 1999).

The electron impact ionization is the main ionization process occurring in the laser-produced plasma. It is well-known (Abdallah et al., 2001; 2003; Zhidkov et al., 2001; Hansen et al., 2002; Auguste et al., 2001; Fournier et al., 2003; Moribayashi et al., 2001) that in plasma produced by laser pulses with intensities higher than $10^{16} \mathrm{~W} / \mathrm{cm}^{2}$, hot electrons with energies about a few $\mathrm{keV}$ are generated very efficiently. However, the rates of electron impact ionization are proportional to $n^{3}$ ( $n$ is the principal quantum number of the ionizing electrons). It means that hot electrons preferentially ionized outer-shell electrons rather than inner-shell ones. Thus, during hot electron impact ionization hollow ions are formed, but their abundance is sufficiently lower than the abundance of usual ions. For example, it was shown by Moribayashi et al. (2001) that the $\mathrm{K}^{2}$-hollow ion population is only about $1 \%$ relative to states with single inner-shell vacancy even for laser intensity of $I \sim 10^{18} \mathrm{~W} / \mathrm{cm}^{2}$.

When an X-ray photon interacts with an iron, the electron removal cross-section (the photoionization cross section) is approximately proportional to $n^{-5}$, i.e., the photon incident on the ion will remove electrons with overwhelmingly high probability from the innermost shell whose ionization energy does not exceed the photon energy. It means that in contrast to the optical laser radiation interaction with the 
matter, the absorption of X-ray radiation directly causes the formation of hollow ions. Currently, the process of hollow ion production by $\mathrm{X}$-ray radiation is becoming more topical in connection with the advent of high-power X-ray lasers, both plasma-based (Elton, 1990; Rocca, 1999; Daido, 2002; Suckewer \& Jaegle, 2009) and free-electron lasers (Emma et al., 2010; Vinko et al., 2012; Mimura et al., 2014). With such lasers, the energy input process, unlike that in the case of interaction of optical laser radiation with matter, is directly related to hollow ion production.

$\mathrm{X}$-ray radiation capable of producing hollow ions may be emitted by the plasma itself. This effect should be most pronounced when the plasma is strongly inhomogeneous and there are regions with substantially different temperatures. In this case, the radiation generated in the hot region will produce hollow ions in the cold region where the electron temperature is not high enough to ionize the ion outer-shells (Abdallah et al., 2000; Colgan et al., 2010). Another very important X-ray source that can produce hollow ions has recently been experimentally realized (Colgan et al., 2013; Pikuz et al., 2013; Hansen et al., 2014). It was demonstrated when the optical laser radiation with an intensity of $>10^{20}$ $\mathrm{W} / \mathrm{cm}^{2}$ interacted with thin foils. In such experiments, fast electrons are effectively generated and begin to oscillate through the foil. Due to radiation dominant regime effect (Zhidkov et al., 2002; Nakamura et al., 2012; Ridgers et al., 2012; Pandit \& Sentoku, 2012) such oscillating electron beam produces $\mathrm{X}$-rays with an extremely high intensity of $10^{19} \mathrm{~W} / \mathrm{cm}^{2}$. It should be noted that the intensity of such broadband radiation source exceeds the intensity of monochromatic and coherent X-ray free electron laser beam (Vinko et al., 2012).

This paper is a short review on the possible applications of the hollow ion spectra for the diagnostics of low and hightemperature solid density plasma created by intensive laser and particle beams. Because of the hollow ion spectra features are defined mainly by the mechanisms of their excitation, we will consider the various types of the multicharged ion plasma, where different excitation processes are important.

\section{HIGH-TEMPERATURE PLASMA CREATED BY EXTERNAL INTENSIVE X-RAY RADIATION}

To use the hollow ion spectra for diagnosing of the plasma affected by strong X-ray radiation have been proposed recently in the papers by Vinko et al. (2012) and Colgan et al. (2013). It should be noted that the properties of such high energy density plasma are under increasing scrutiny in recent years due to their importance to our understanding of stellar interiors, the cores of giant planets (Chabrier, 2009; Kalman, 2010), and the properties of hot plasma in inertial confinement fusion devices (Atzeni \& Meyer-Ter-Vehn, 2004). These high-energy density systems are relevant to study photoionized plasmas found in active galactic nuclei and X-ray binaries (Behar et al., 2001).
With irradiation by X-rays, electrons in the inner-shells are ionized first, which creates atoms or ions with empty internal electron shells, i.e., hollow atoms or ions. Note that the powerful new X-ray sources, namely, free electron lasers, are now available and could provide laser intensities larger than $10^{17}$ $\mathrm{W} / \mathrm{cm}^{2}$ for creation in laboratory conditions of novel states of matter that also must show clear signatures of hollow ions formation.

Recently, it was shown that strong X-ray radiation can also be formed with long wavelength laser fields, if the laser intensity is sufficiently large and approaches the radiation dominant regime (Zhidkov et al., 2002). In this case, a highintensity laser rapidly ionizes valence electrons from a thin target through field ionization (Zhidkov \& Sasaki, 2000). Such electrons are accelerated in the strong electric field of the laser and can quickly reach very high energies $(>10 \mathrm{MeV})$ if the laser is sufficiently intense. During the interaction of an intense laser pulse with a thin foil some of these hot electrons oscillate through the foil (Nakamura et al., 2012; Antici et al., 2007), due to reflection by the fields on each surface, known as refluxing. These electrons radiate X-rays via Thomson scattering of the incident and scattered laser light and via Bremsstrahlung through the strong plasma field at the foil surfaces. It was estimated by Colgan et al., (2013) that both of such processes can produce $\mathrm{X}$-rays in the few $\mathrm{KeV}$ range and at intensities of around $10^{19}$ $\mathrm{W} / \mathrm{cm}^{2}$, when conventional optical laser intensity exceed $10^{20} \mathrm{~W} / \mathrm{cm}^{2}$. The proposed concept (see Fig. 2) was tested at the Vulcan Petawatt Laser Facility at Rutherford Appleton

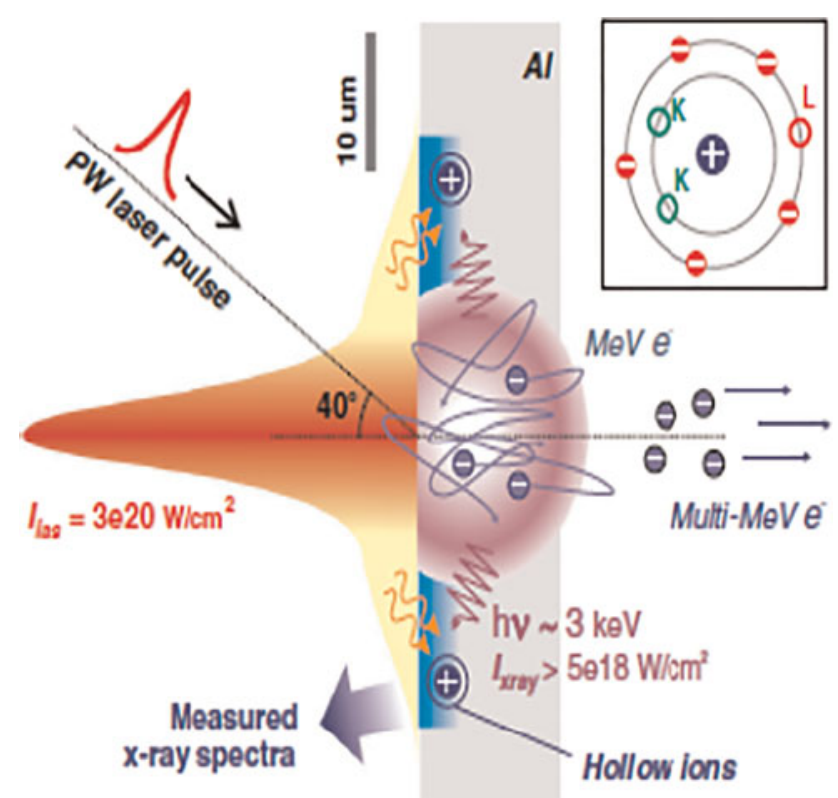

Fig. 2. A schematic diagram of hollow atoms formation by the ultra intense optical laser pulse (Colgan et al., 2013). Laser field of $>10^{20} \mathrm{~W} / \mathrm{cm}^{2}$ in central area of the focal spot accelerates $\mathrm{MeV}$ and multi-MeV electrons from a target and produces bright $\mathrm{X}$-ray radiation range via Thomson scattering and Bremsstrahlung processes. In turn, X-ray photons of $\mathrm{keV}$ energies create hollow atoms in outer area of a target. 
Laboratory. Details of the experiments are described by Colgan et al. (2013). Note here that when in such experiments the maximum laser intensity of $3 \times 10^{20} \mathrm{~W} / \mathrm{cm}^{2}$ was achieved, laser pulse duration was 0.7 ps, and the laser contrast was about $10^{9}$.

The spectra were measured in this paper using a focusing spectrometer with spatial resolution (FSSR) equipped with spherically bent mica crystals (Faenov et al., 1994; Skobelev et al., 1995; Blasco et al., 2001). FSSR was tuned to observe radiation in the wavelength range from 7.0 to $8.4 \AA$ containing the $\mathrm{K}$-shell spectra from multi-charged and neutral $\mathrm{Al}$ ions. The spectra were acquired from the front, laser irradiated surface, at $45^{\circ}$ to the target normal. X-ray spectra from pure aluminum foils of 1.5 and $20 \mathrm{mkm}$ thicknesses were investigated at different laser pulse energies.

As shown in Figure 3, the reduction of the laser pulse energy from $160 \mathrm{~J}$ to $64 \mathrm{~J}$ leads to remarkable changes in the spectra. For the lower laser energy case, little emission was observed, with prominent peaks just below 7.8 and 7.2 $\AA$ corresponding to $\mathrm{He}_{\alpha}$ and $\mathrm{Ly}_{\alpha}$ transitions. In the full laser energy case, the spectra are dramatically different. The "usual" $\mathrm{He}_{\alpha}$ and $\mathrm{Ly}_{\alpha}$ lines are dominated by significant emission between 7.3 and $7.7 \AA$ (which was identified as arising from KK hollow atoms) and between 7.9 and $8.3 \AA$ (which was associated with emission from KL hollow atoms). These spectra differ notably from observations previously made with high contrast optical lasers (See for
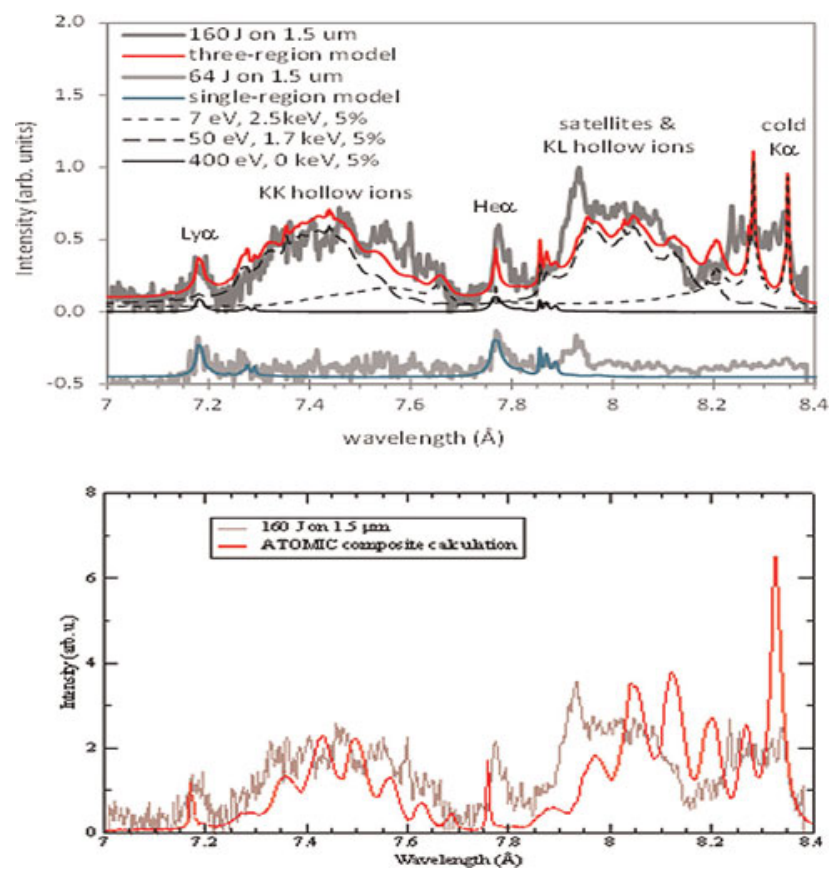

Fig. 3. Upper panel: Experimental spectra measured at Vulcan PW laser with $\mathrm{Al}$ foil targets of $1.5 \mu \mathrm{m}$ thickness and laser energies of 160 or $64 \mathrm{~J}$ (Colgan et al., 2013; Hansen et al., 2014). Comparison of SCRAM modeled results, which fits to experimental spectra at two different laser intensities. Lower panel: ATOMIC comparison to the experimental spectra for the $160 \mathrm{~J}$ laser interaction with $\mathrm{Al}$ foil target of $1.5 \mu \mathrm{m}$ thickness example, Andiel et al., 2002a; 2002b) and rather high laser intensities about $10^{17} \mathrm{~W} / \mathrm{cm}^{2}$. The new emission at wavelengths lower than the $\mathrm{He}_{\alpha}$ line is due to the radiative decay from the L-shell to the $\mathrm{K}$-shell within ions that have a double K-shell vacancy, as well as several L-shell vacancies, quite distinct from earlier studies of hollow atoms (McPherson et al., 1994), which involved LM transitions. Possible hollow atom configurations are shown schematically in Figure 1 and the inset of Figure 2. The substantial decrease in the yield of hollow ion spectral lines occurs, consistent with the strong dependence of the X-ray yield on laser intensity (Zhidkov \& Sasaki, 2000; Zhidkov et al., 2002).

Emission arises from many ion stages of $\mathrm{Al}$, from $\mathrm{Al}$ III through $\mathrm{Al} \mathrm{X}$. For emission to be observed from such ion stages at these high field intensities, implies that the electron density remains high (greater than $10^{23} \mathrm{~cm}^{-3}$ ), so that threebody recombination drives the ions back toward the neutral stage. The emission in the longer wavelength range (7.9 to $8.3 \AA$ ) results from a similar radiative decay process, except that in this case, the initial state has only one K-shell vacancy. This emission occurs at slightly later times, when the electron density is smaller and the radiation field has significantly decreased. Such transitions are less energetic and so occur at longer wavelengths. Refluxing of fast electrons, which are responsible for X-ray radiation, between the target's front and rear occurs only in thin foils and vanishes in thick ones. This is the primary reason for the difference of spectra observed by Colgan et al. (2013) for the 1.5 and $20 \mathrm{mkm}$ foils.

It is clear that the large energy deposited in the present experiment leads to a quite different physical picture than that used for lower energy laser experiments. The intense Vulcan laser rapidly ionizes valence electrons from the $\mathrm{Al}$ target through field ionization (Zhidkov \& Sasaki, 2000). These electrons are accelerated in the strong electric and magnetic fields of the laser, and the ponderomotive electron energy (Gavrila, 1992) in a laser field at an intensity of $>10^{20} \mathrm{~W} / \mathrm{cm}^{2}$, approaches to $10 \mathrm{MeV}$. The thin $\mathrm{Al}$ target is essentially transparent to these highly-relativistic electrons (Zhidkov \& Sasaki, 2000), but the electrons quickly lose energy through plasma bremsstrahlung (Nozaw et al., 1998) and (non-linear) Thomson scattering (Sarachik \& Schappert, 1970; Landau \& Lifshitz, 1961). The scaling for the radiation power $P$, with the laser amplitude $a_{0}=$ $e E_{\mathrm{L}} / m c \omega\left(E_{\mathrm{L}}\right.$ is the electric field strength, $\omega$ is the field frequency, $m$ is the electron mass, and $c$ is the speed of light) can be estimated with the use of radiation friction force (Zhidkov et al., 2002).

These X-rays interact with the $\mathrm{Al}$ ions and atoms in the target by interacting with electrons in the inner atomic shells and ejecting multiple $\mathrm{K}$ and L-shell electrons. Innershell electrons are preferentially ionized by high-energy photons so that the excess recoil momentum can be absorbed by the nucleus. High-energy photons are much more efficient at removing K-shell electrons than electrons of similar energies (Skobelev et al., 2012). A previous measurement (Evans 
et al., 2005), which used similar laser intensities to the present measurement but a much thicker target, found that electron pumping of hollow ions was the main ionizing mechanism. In this case, it was found that the generation of hollow ions was not efficient.

To produce a sufficient number of the KK hollow ions, the photoionization must be the main channel of the autoionization state decay. That means the condition $\left(\mathrm{I}_{\mathrm{X}-\text { ray }} /\right.$ $\hbar \omega) \sigma^{\mathrm{ph}}>\Gamma$ must be satisfied (here $I_{\mathrm{X} \text {-ray }}$ is the $\mathrm{X}$-ray flux, $\hbar \omega$ is the energy of X-ray photons, $\sigma^{\mathrm{ph}}$ is the photoionization cross-section, and $\Gamma$ is the probability of autoionization). For Al XII ion, the K-shell photoionization cross-section at $\hbar \omega \sim$ $2 \mathrm{keV}$ is about $5.5 \times 10^{-20} \mathrm{~cm}^{2}$, probabilities of autoionization decay are about $10^{15} \mathrm{~s}^{-1}$. Therefore the $\mathrm{X}$-ray flux greater than $10^{19} \mathrm{~W} / \mathrm{cm}^{2}$ must be realized to provide such intense hollow ions spectra. We believe that radiation dominant regime is the most suitable mechanism for production so bright X-ray source.

$\mathrm{X}$-ray spectra of hollow atom radiation were analyzed by performing atomic kinetics calculations using the ATOMIC code ((Mazevet \& Abdallah, 2006; Colgan et al., 2010; 2011; 2013) and the hybrid structure Spectroscopic Collisional-Radiative Atomic Mode (SCRAM) (Hansen et al., 2007; 2014). These codes include all relevant atomic processes, that is, photoionization, collisional ionization, autoionization, collisional and radiative excitation and de-excitation, and all recombination processes. Preliminary time-dependent calculations using a simplified atomic model were performed to determine the bulk plasma conditions. They show that, at the high electron densities under consideration here, the system reaches steady-state very quickly. As it was shown by Hansen et al. (2014) for correctly modeling of the exotic plasma created experimentally, it is necessary to include over 21000 atomic configurations, representing $\mathrm{Al}$ ions in which up to five electrons have been removed from the $\mathrm{K}$ - and L-shells and placed in excited states. Multiply excited states were found to be important in accurately reproducing the observed emission spectra. In particular, it was demonstrated that the radiative decay from the L- to K-shell in any one specific configuration results in a relatively weak line, but that the very large number of configurations with different combinations of spectator electrons in excited states, in which this radiative transition occurs, allows the observed transition to become prominent.

Performed detailed steady-state calculations at various plasma conditions allowed to compute (Colgan et al., 2013; Pikuz et al., 2013; Hansen et al., 2014) the emission spectrum as a function of electron temperature (including non-Maxwellian effects such as hot electron tails) and density. Additionally, opacity effects are included via escape factors. Influence of radiation temperature was included assuming that the radiation field seen by the plasma was a Planckian distribution in the keV range. Typical dependencies of spectra radiation form such parameters are presented in Figures 4-7. As it is seen from Figure 4 no emission lines

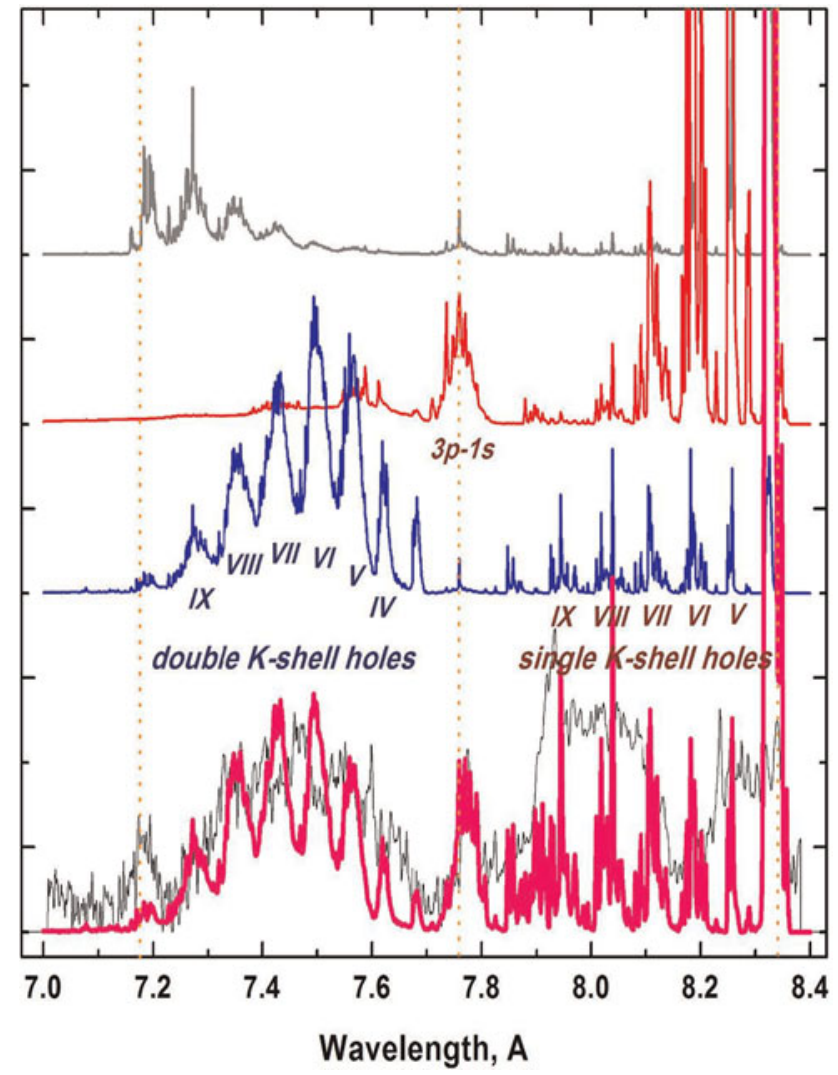

Fig. 4. Aluminum K-shell spectra calculated (Colgan et al., 2013) by the ATOMIC code. Calculations are made at plasma conditions: bulk $T_{\mathrm{e}}=55$ $\mathrm{eV}, \mathrm{Ne}=3 \mu \times 10^{23} \mathrm{~cm}^{-3}, 5 \%$ of $5 \mathrm{keV}$ hot electrons, $T_{\text {rad }}=3 \mathrm{keV}$. The inset shows the ion charge distribution for the combined calculation (pink line in both panels) that includes the radiation field and hot electrons. Experimental spectra were measured for $\mathrm{Al}$ foil targets of $1.5 \mu \mathrm{m}$ thickness and with laser energies of $160 \mathrm{~J}$.

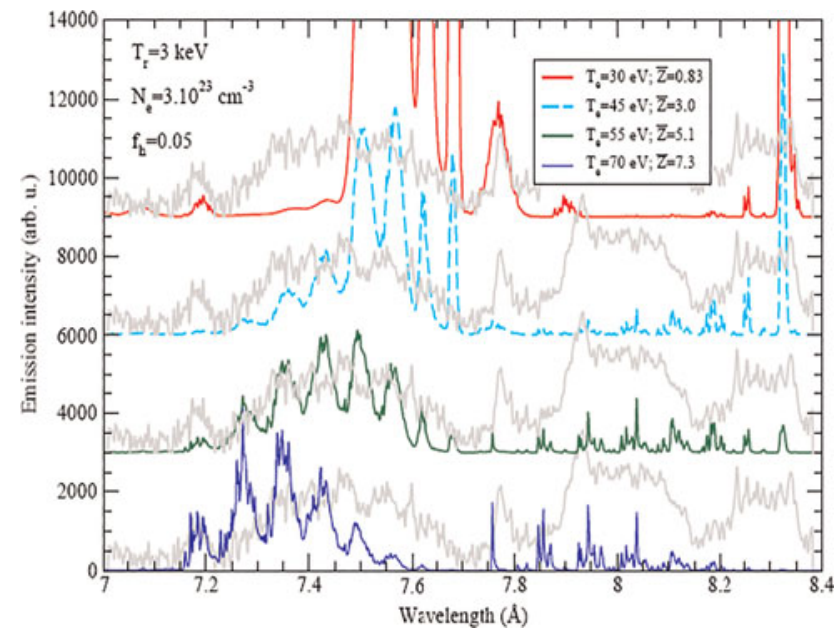

Fig. 5. Aluminum K-shell spectra calculated by the ATOMIC code (Pikuz et al., 2013). ATOMIC calculations are made for four different electron temperatures and the following other plasma conditions: Ne-3 $3 \times 10^{23} \mathrm{~cm}^{-3}, 5 \%$ of $5 \mathrm{keV}$ hot electrons, $T_{\mathrm{rad}}=3 \mathrm{keV}$. Spectra measured in Colgan et al. (2013) is shown as the grey line. 


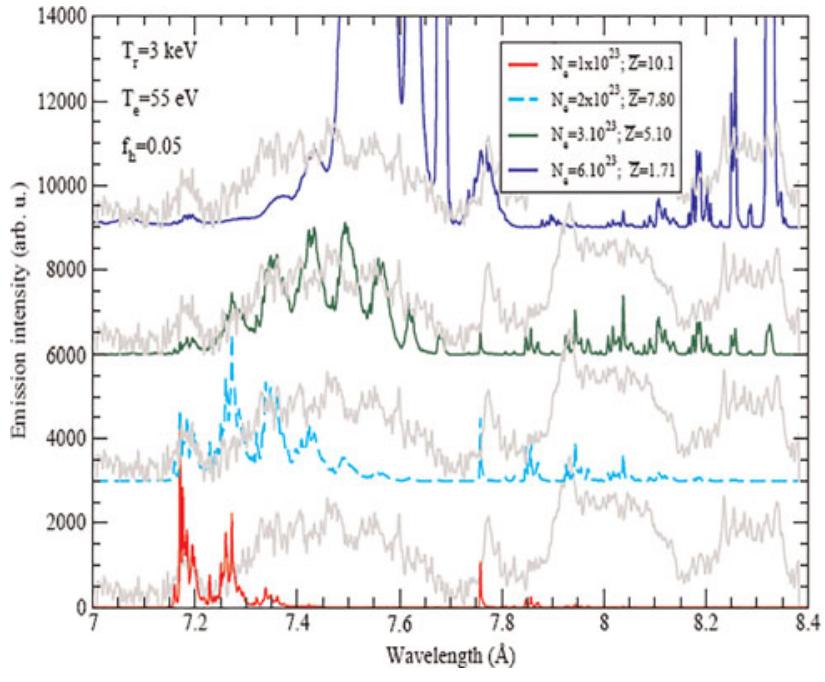

Fig. 6. Aluminum K-shell spectra calculated by the ATOMIC code (Pikuz et al., 2013). ATOMIC calculations are made for four different electron densities and the following other plasma conditions: $T_{\mathrm{e}}=55 \mathrm{eV}, 5 \%$ of $5 \mathrm{keV}$ hot electrons, $T_{\mathrm{rad}}=3 \mathrm{keV}$. The inset shows the ion charge distribution for the combined calculation (pink line in both panels) that includes the radiation field and hot electrons. Spectra measured in Colgan et al. (2013) is shown as the grey line.

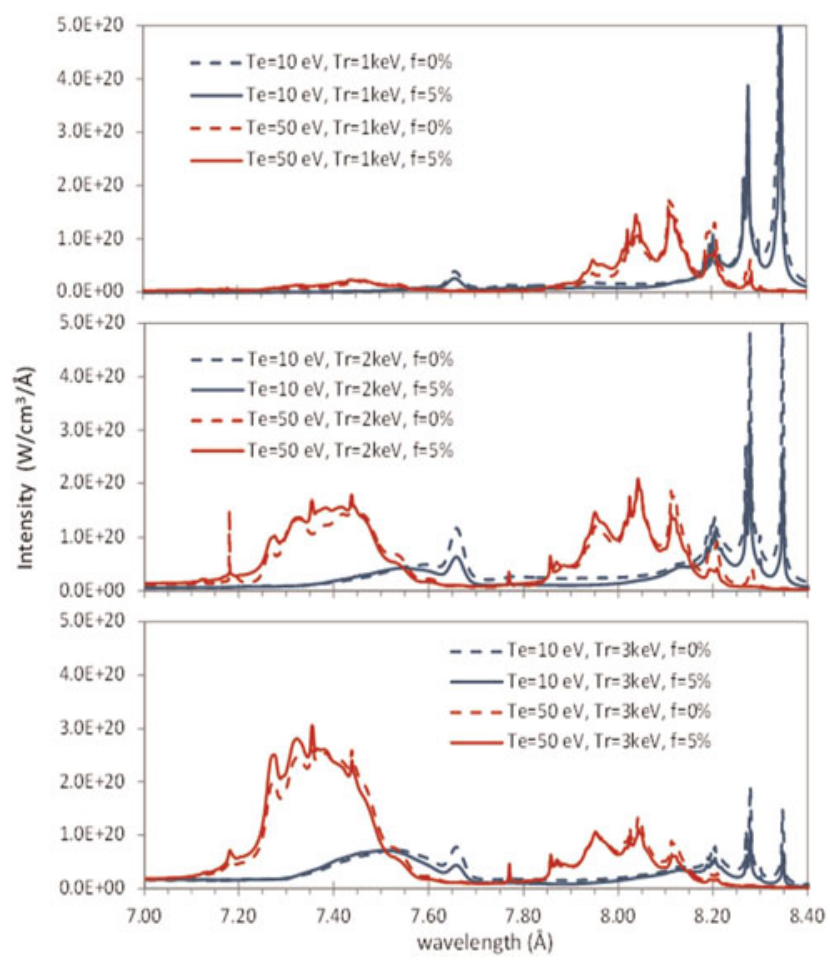

Fig. 7. Calculated (Hansen et al., 2014) optically thin emission from the SCRAM model showing dependence of emission on $\mathrm{Te}, \mathrm{Tr}$, and hot electron fraction, assuming a $5 \mathrm{keV}$ Maxwellian hot electron distribution.

due to KK hollow ions are observed, particularly in the region between 7.2 and $7.7 \AA$, in the case of absence external radiation field. Figure 4 obviously demonstrated that the incorporation in modeling radiation field leads to dramatic effect. The blue line shows the effect of radiation on the calculation while omitting the hot-electron tail. Modeling shows that the hot electrons do excite hollow ions, but provide only a small part of the measured intensity of the hollow ion spectral lines. It was also found (see Fig. 7) that at a radiation temperature of $1 \mathrm{keV}$ only a few spectral features exist in the region 7.2-7.6 A. However, for $T_{\mathrm{r}}=2 \mathrm{keV}$, we see a dramatic change; a series of intense lines are now prominent. As we move to $T_{\mathrm{r}}=3 \mathrm{keV}$ the intensity of these features is modified somewhat (and is in better agreement with the observed spectrum).

Figures 4-7 demonstrate strong dependence of emitted spectra intensities from all plasma parameters. It is necessary to stress that intensity of KK and KL hollow ions spectra are the most pronounced for bulk electron temperatures around $10-100 \mathrm{eV}$ and electron densities about $10^{23} \mathrm{~cm}^{-3}$ or higher. At the same time, for observation of intensive, KK hollow ions spectra high radiation temperature are crucial parameters. Presented results of theoretical modeling allow concluding that if $X$-ray spectra with high $(\lambda / \Delta \lambda$ more than 2000) spectral resolution are observed, it is allowed to determine parameters of warm, dense plasma with rather high accuracy.

Comparisons of modeled by SCRAM and by ATOMIC codes spectra with experimental one obtained at laser energy $160 \mathrm{~J}$ are presented in Figure 3. It is clearly seen that both codes model experimental spectra rather well. At the same time it was shown that it is impossible to model all lines intensities using only one set of plasma parameters. From Figure 3 (upper panel) we could conclude that the emission comes from three almost equally weighted regions, all assumed to be at solid density. The first region includes the entire depth of the $1.5 \mathrm{mkm}$ target at a relatively cold thermal temperature $(7 \mathrm{eV})$, with $5 \%$ hot electrons and a $2.5 \mathrm{keV}$ radiation field. This region contributes the cold $\mathrm{K}_{\alpha}$ and the $\mathrm{KL}$ and $\mathrm{KK}$ emission from near-Ne-like ions. The second region has a higher thermal temperature of 50 $\mathrm{eV}$ and a lower radiation temperature of $1.7 \mathrm{keV}$, and is responsible for the KL and KK emission from mid-L-shell ions. Finally, there is a hotter region with $T e=400 \mathrm{eV}$ and $5 \%$ hot electrons with no radiation field that includes only about a tenth of the original target thickness, but with a larger weighting factor that could be attributable to a larger area or longer duration. The spectrum measured from the $64 \mathrm{~J}$ laser pulse incident on a $1.5 \mathrm{mkm}$ target is also shown in Figure 3 (upper panel) and it is quite different compared with the spectra emitted by plasma irradiated by laser with energy $160 \mathrm{~J}$. This spectrum does not have significant hollow-ion emission and is reasonably well fit by the high-temperature region with no radiation field. Such strong change in spectra intensity and processes responsible for it production is consistent with the mechanism for radiation production due to radiation dominant regime, which were discussed above and was proposed in Colgan et al. (2013). 
So, we see that hollow-ion emission from radiationdominated hot, dense plasmas provides a new opportunity for diagnosing high-intensity X-ray radiation fields interaction with warm dense matter. However, constructing adequate non-LTE atomic models remains a challenge, since there remains uncertainty in the ionization potential depression at these conditions, configuration interaction plays a significant role in the structure of the emission, and multiply excited states with many holes in both valence and inner shells can lead to enormous structural and computational complexity. Developing atomic kinetics model that systematically and self-consistently accounts for all these effects is state-of-the-art for atomic and plasma physics today.

\section{HIGH-TEMPERATURE PLASMA WITH ENHANCED ROLE OF THE CHARGE-EXCHANGE PROCESSES}

When a gas is utilized as a laser target, there are favorable conditions for the penetration of multi-charged ions, which are generated in the focal region, into peripheral regions containing primarily low-charged ions, and even neutral atoms of the gas. This has the consequence that the charge exchange processes may give rise to an efficient population of the states of hollow ions.

This effect was first discovered and explained by Rosmej et al. (1999), which was dedicated to the experimental and theoretical investigation of intense femtosecond laser pulses with high-pressure nitrogen. The experiments of Rosmej et al. (1999) were performed on the titaniumsapphire laser of the Saclay Research Center (France). Laser pulses with a linear $p$-polarization were focused onto a pulsed nitrogen gas target. The atomic number density was amounted to $1.5 \times 10^{19} \mathrm{~cm}^{-3}$ for the highest pressure in the valve equal to 20 bar. The pulse energy was $750 \mathrm{~mJ}$, the laser contrast ratio was $10^{5}$, and intensity was equal to $10^{19} \mathrm{~W} / \mathrm{cm}^{2}$. Under this intensity, the optical field ionization could take place up to the instant of formation of bare nitrogen nuclei (Ammosov et al., 1986). The space-resolved X-ray spectra of nitrogen ions were recorded using a spectrograph with a spherically mica crystal (Skobelev et al., 1995; Faenov et al., 1994) bent to a radius of $150 \mathrm{~mm}$. The observed spectra contained very broad structures about the resonance lines of the H-like ion (see Fig. 8).

Rosmej et al. (1999) hypothesized that these structures arise from radiative transitions from the highly excited $\mathrm{nln}_{0} \mathrm{l}_{0}$ states of hollow He-like nitrogen ions. Atomic calculations performed using the multi-configuration HartreeFock method with the inclusion of relativistic corrections for hollow configurations $\left(3151_{0}, 4151_{0}, 5151_{0}, 3161_{0}, 4161_{0}\right.$, $5161_{0}, 6161_{0}, 3171_{0}, 4171_{0}, 5171_{0}, 6171_{0}, 7171_{0}, 3181_{0}, 4181_{0}$, $5181_{0}, 6181_{0}, 7181_{0}$, and $8181_{0}$ ) confirmed this assumption, at least for the wavelengths of the observed spectral peaks. It turned out that the spectra of the structures listed above are really quite broad because of the mutual overlap of a huge number of closely spaced transitions and lie in the

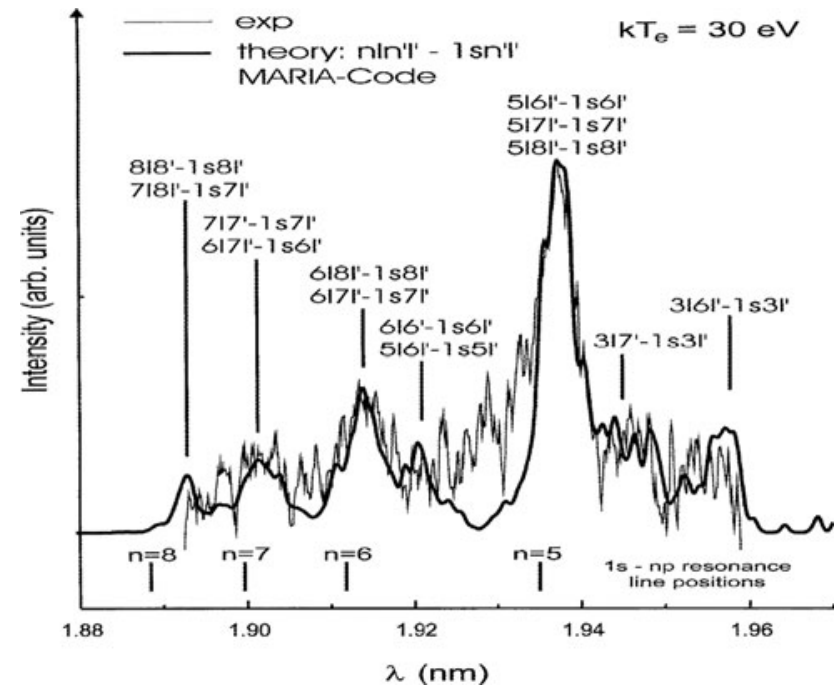

Fig. 8. Comparison of the experimental spectrum of the nitrogen target with the spectrum calculated using the Maria code (Rosmej et al., 1999).

spectral region of interest. Furthermore, the total emission spectrum of these hollow ion states provides a qualitatively and even quantitatively good description of the experimental spectrum (a comparison is given in Fig. 8) with the exception of the H-like ion resonance lines themselves. This signifies that, in this case, the hollow ion states for some reasons are anomalously densely populated in comparison with the singly excited states of hydrogen-like ions. To explain this anomalous behavior of level populations, Rosmej et al. (1999) proposed a physical mechanism involving charge exchange in collisions of multi-charged and lowcharged nitrogen ions. The scenario of this mechanism is as follows.

After the optical field ionization of $\mathrm{N}_{2}$ molecules to the state of bare nuclei, they penetrate the surrounding gas that contains neutral atoms or singly and doubly ionized ions. The reason is that the laser intensity decreases rapidly with distance from the central spot, and at distances of about 30 $\mathrm{mkm}$ from the focused point, the laser field cannot ionize even $\mathrm{N}_{2}$ molecules. We note, however, that $\mathrm{N}_{2}$ molecules, even though far away from the central spot, may be destroyed by sufficiently high-energy particles (photons and electrons) generated in the central region. That is why, in accordance with Rosmej et al. (1999), it will be assumed that the nuclei collide with $\mathrm{N}^{+0}, \mathrm{~N}^{+1} \ddagger$ and $\mathrm{N}^{+2} \ddagger$ ions, resulting in the following single- or double-electron charge-exchange reactions $\quad \mathrm{N}^{6+}+\mathrm{N}^{0} \rightarrow \mathrm{N}^{5+}(\mathrm{nl})+\mathrm{N}^{1+}, \quad \mathrm{N}^{6+}+\mathrm{N}^{1+} \rightarrow$ $\mathrm{N}^{5+}(\mathrm{nl})+\mathrm{N}^{2+}, \quad \mathrm{N}^{6+}+\mathrm{N}^{2+} \rightarrow \mathrm{N}^{5+}(\mathrm{nl})+\mathrm{N}^{3+}, \quad \mathrm{N}^{6+}+$ $\mathrm{N}^{0} \rightarrow \mathrm{N}^{4+}\left(n \ln \mathrm{l}^{\prime}\right)+\mathrm{N}^{2+}, \quad \mathrm{N}^{6+}+\mathrm{N}^{1+} \rightarrow \mathrm{N}^{4+}\left(n \ln \mathrm{l}^{\prime}\right)+$ $\mathrm{N}^{3+}, \mathrm{N}^{6+}+\mathrm{N}^{2+} \rightarrow \mathrm{N}^{4+}\left(\right.$ nln $\left.^{\prime} \mathrm{l}^{\prime}\right)+\mathrm{N}^{3+}$. It means that the charge exchange mechanisms do turn out to be most significant in the population of hollow ion states.

One can see from the Figure 8 that comparison of the calculated hollow ion spectra with observed ones allow to measure plasma parameters. For example, in the experiment of 
Rosmej et al. (1999) plasma density was known, and value of $T_{\mathrm{e}}=30 \mathrm{eV}$ was measured by this method.

\section{EMISSION OF HOLLOW IONS SPECTRA IN HIGH-TEMPERATURE PLASMA WITH FAST CHARGED PARTICLES}

It is well-known that in the laser-produced plasma and particular in plasma produced by relativistic laser intensities, a large amount of fast electrons and ions are generated. However, as it was mentioned above, the creation of the hollow ions by charged particle impact ionization is less effective than by X-ray photons. Moreover, the extra high plasma density is needed for the good excitation of the hollow ion states. At the same time in the laser-produced plasma, the electron plasma density usually is limited by critical value, depending on laser wavelength. This limitation is not important only for super short laser pulses (femtosecond or sub-picosecond) and for short wavelength lasers (vacuum-ultraviolet- or $\mathrm{X}$-ray). Therefore, until now, hollow ion spectra were observed practically only in the femtosecond laser-produced plasma in the cases when laser did not produce preplasma due to the rather low laser intensity (Gauthier et al., 1995) or due to very high (above $10^{9}$ ) laser contrast (see, for example, Faenov et al., 1997; 1999; 2009; 2011; 2012; Urnov et al., 1998; Rosmej et al., 2000; Colgan et al., 2008; 2011; Skobelev et al., 2012). For longer laser pulse, hollow ion spectra have been observed where short wavelength eximer $\mathrm{XeCl}$-laser or $2 \mathrm{w}$ of $\mathrm{Nd}$ laser were used (Abdallah et al., 2000; Colgan et al., 2008a; Colgan 2010).

For the plasma created by fast heavy ion beam situation must be better, because in this case the beam energy will be inputted directly in the solid state target and plasma density will be higher (the critical density does not exist in this case). It means that such plasma hollow ions will be created independently on the ion pulse duration (see for example, Moribayashi et al., 2001a). The ion energy for effective ionization of inner atom electrons must be higher than the energy of fast electrons, of course, but the experiments, carried out at the different accelerators, showed that energy of nowadays used heavy projectile ions are enough for ionization of the K-electrons in the different atoms (see, for example, MacFarlane et al., 1993; Rosmej et al., 2002; Rosmej, O. et al., 2003; 2005b; 2005a; Pikuz et al., 2006; Rzadkiewicz et al., 2010; Kawamura et al., 2011; McMahon et al., 2011; Nakano et al., 2012).

To the present time KK hollow ion spectra were used for diagnostics of high-temperature plasma with fast charged particles only in the case of the laser-produced plasma. The observations of the KK-hollow ion spectra (spectra of the ions with two holes in the K-shell) in the plasma heated by heavy ion beam are absent yet. We would like to note that there are no objective reasons for this and consider that such observations in future will allow obtaining important information on heavy ion beam plasma. It should be noted that KL-hollow ions (ions with one hole in the K-shell and one hole in the L-shell) have been observed in aluminum plasma heated by intensive proton beam (Bailey et al., 1990). In the papers by Wang et al. (1993) and MacFarlane et al. (1993) it was shown that $\mathrm{K}_{\alpha}$ satellite lines of aluminum ions observed by Bailey et al. (1990) can be classified into two distinct groups: one involving transitions of type $1 \mathrm{~s} 2 \mathrm{~s}^{\mathrm{r}} 2 \mathrm{p}^{\mathrm{q}}$ $1 s^{2} 2 s^{r} 2 p^{q-1}$, the other $1 s 2 s^{r} 2 p^{q-1} 31-1 s^{2} 2 s^{r} 2 p^{q-2} 31$. The second group is just KL-hollow ion configurations.

In the case of the laser-produced plasma, the hollow ion spectra will be prominent in the densest regions, while emission spectra of the hot low dense plasma are being caused by usual spectral transitions. Just for diagnostics of these regions, the hollow ion spectra are the most suitable. For example, Colgan et al. (2011) and Faenov et al. (2011; 2012) have suggested the use of such spectra to diagnose the early stage of the heating of argon clusters by a femtosecond laser pulse. Experiments were carried out at the Xtreme Light III (XL-III) laser facility at the Institute of Physics, Chinese Academy of Sciences. The laser pulse has energy up to $1 \mathrm{~J}$, duration is about $100 \mathrm{fs}$, and contrast is about $10^{10}$. A laser beam was focused onto a pulsed gas-cluster argon target. The laser energy flux density reached $q_{\text {las }}=2.6 \times 10^{19} \mathrm{~W} / \mathrm{cm}^{2}$. The pressure of the gas supplied to the nozzle was optimized to increase the yield of clusters with a radius of $R_{\mathrm{cl}} \sim$ $0.15 \mu \mathrm{m}$. According to the calculations of Boldarev et al. (2004; 2006), a cluster diameter of $0.3 \mu \mathrm{m}$ and a maximum atomic density of $N_{\mathrm{Ar}} \sim 1.5 \times 10^{20} \mathrm{~cm}^{-3}$ at a distance of $1 \mathrm{~mm}$ from the nozzle edge are achieved at a gas pressure of 70 bar. The experiments indicated that the maximum $\mathrm{X}$-ray emission was observed at this gas pressure.

In our previous experiments, the spectra of the argon plasma were studied in the laser-droplet interaction (the size of cluster was about $1 \mu \mathrm{m}$ ) regime (see Fig. 9). Dependences of intensities of argon spectra from contrast and pulse duration are presented in Figure 9 and demonstrated that in the case of high laser contrast and very short laser pulse duration the strong KL hollow ions spectra are radiated. It was found that various stages of the evolution of the produced plasma contribute to the observed time-integrated $\mathrm{X}$-ray emission spectrum. The long-wavelength part of the spectrum shown in Figure 9 is formed at the initial heating stage by the densest regions heated not too strong, whereas the short-wavelength part is formed by the less dense, hotter plasma at the much later stage. Since the size of clusters in these experiments was about $1 \mu \mathrm{m}$, i.e., was much larger than the thickness of the skin layer, the heating of the cluster was non-uniform. When its surface layer had been already heated to a high temperature and began to expand, the inner part still remained cold and immobile.

In the case under consideration, all parts of the cluster will be heated simultaneously and the plasma created at the heating stage will be much more uniform. Using developed stationary kinetic model and the atomic constants calculated for argon ions (Colgan et al., 2011) the emission spectrum corresponding to this stage of the plasma evolution were calculated. It was shown that until the cluster does not 

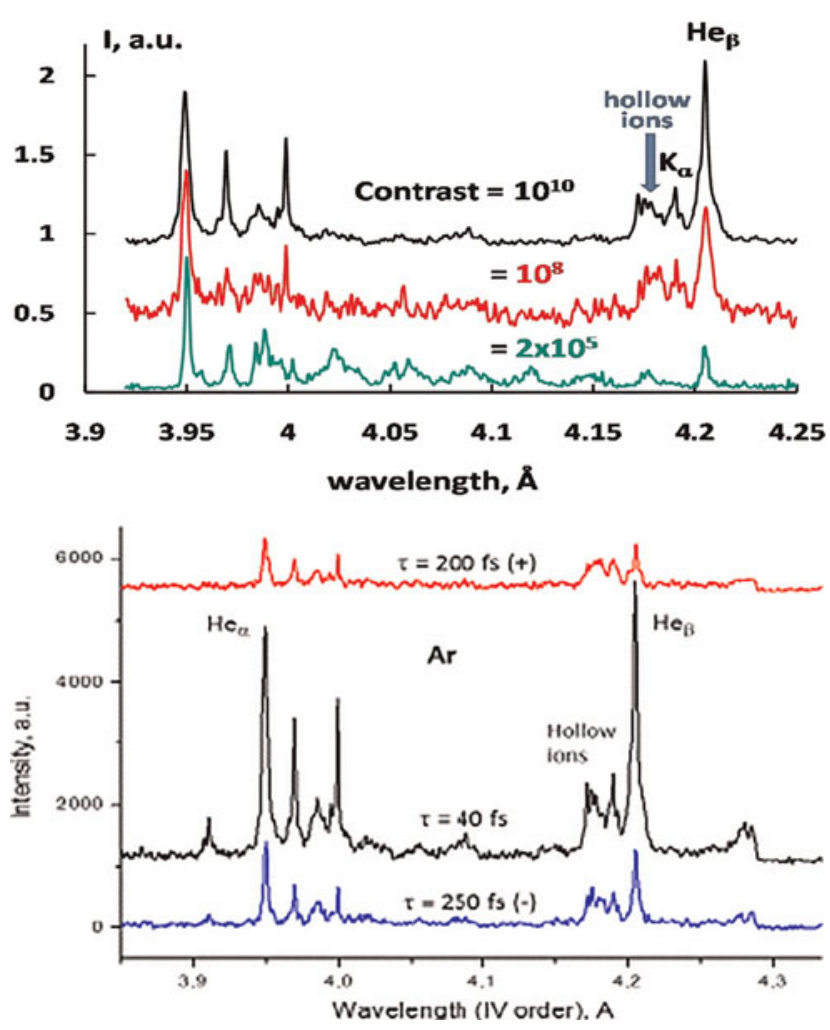

Fig. 9. Typical traces of the spectra in the wavelength range 3.9-4.3 $\AA$ emitted by large size Ar clusters, irradiated by ultra-intense laser radiation (Colgan et al., 2011; Faenov et al., 2012): Upper panel: dependence from different laser contrast. Bottom panel: dependence from different pulse durations.

noticeably expand (i.e., until time $T_{\exp } \sim R_{\mathrm{cl}} / v_{\text {ion }} \sim 150$ fs at a typical value of $v_{\text {ion }} \sim 10^{8} \mathrm{~cm} / \mathrm{s}$ ), its atomic density can be treated as constant $N_{\mathrm{Ar}}=2.1 \times 10^{22} \mathrm{~cm}^{-2}$. In this case, the spectrum will depend on only one parameter, i.e., the electron temperature $T_{\underline{\underline{e}}}$ of the plasma, because its electron density (and the ionization degree) will be determined by the balance equations. In Figure 10, spectra calculated for various $T_{\mathrm{e}}$ and $N_{\mathrm{e}}$ according to this model, which include into the kinetic equations also the influence of the $1 \%$ fraction of hot electrons with a mean energy of $7 \mathrm{keV}$, are presented.

The spectral range shown in Figure 10 includes three broadspectral structures with the average wavelengths of 4.11-4.13, 4.14-4.15, and 4.18-4.20 A. Each of these structures can generally consist of a number of various spectral transitions both well-known transitions $\left(\mathrm{K}_{\alpha}\right.$ lines and dielectronic satellites) and transitions in hollow ions or hypersatellites.

It can be seen from Figure 10 that an increase in the temperature of the plasma is accompanied by a noticeable change in the emission spectrum. To demonstrate the contribution to the emission spectrum from radiative transitions of various types, calculations disregarding transitions in hollow ions were performed by Faenov et al. (2011) and results are presented in Figure 10 (middle panel). These calculations showed that, for low temperatures below $50 \mathrm{eV}$, the emission spectrum almost completely consists of lines of hollow ions. At a

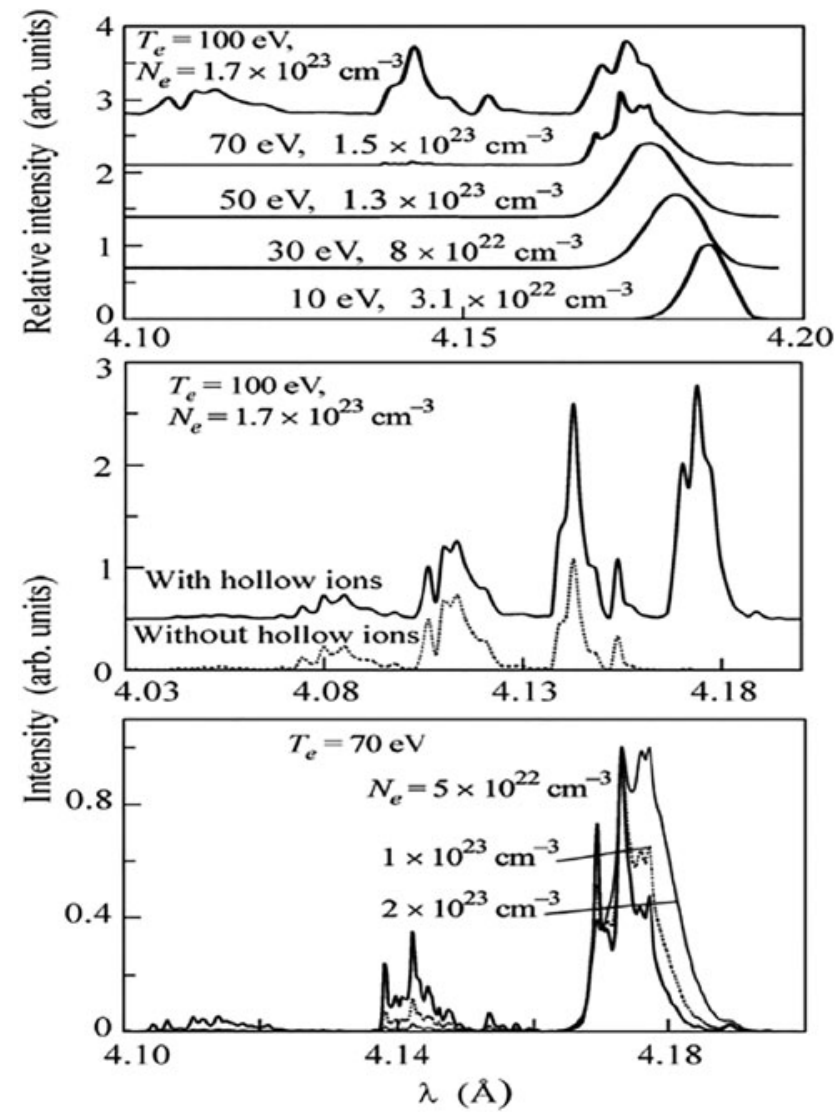

Fig. 10. Emission spectra of Ar plasma calculated (Faenov et al., 2011) in the stationary kinetic model: for various electron temperatures (upper panel); with and without contribution of hollow ions (middle panel); spectra at a fixed temperature but for different electron densities (bottom panel).

temperature of $100 \mathrm{eV}$, hollow ions completely determine the right spectral structure, making an approximately 50\% contribution to the intensity of the central structure, and could be recognized in the left structure (see Fig. 10). It means that the emission of clusters at low temperatures is almost completely determined by transitions in hollow ions. It should be stressed that their contribution in the longestwavelength part of the spectrum under consideration is noticeable even for the high-temperature plasma. It can be seen also from Figure 10 (bottom panel) that an increase in the electron density leads to a decrease in the plasma ionization degree. This is obvious because an increase in the density is accompanied by a fast increase in the collisional recombination rate and by a transition from the coronal ionization equilibrium to the Saha equilibrium. Note that the effect of an increase in $N_{\mathrm{e}}$ on the emission spectrum is similar to that of a decrease in $T_{\mathrm{e}}$ because, in both cases, the plasma ionization degree decreases. Thus, we could conclude that the spectra of hollow ions can be used for the diagnostics of the heating of clusters at the stage of the action of the main laser pulse.

If a high intensive femtosecond pulse interacts with clusters and laser contrast is not large enough, the laser prepulse may destroy cluster and the main pulse will interacts with 
plasma not with dense cluster core. Therefore, development of the diagnostic methods allowing to directly obtain information about the cluster destruction by laser prepulses is very important. In paper by Faenov et al. (2012), it was shown that the key information regarding the existence (or non-existence) of the dense cluster core at the moment of the main laser pulse arrival can be obtained by means of the observation of the hollow ion spectra. Really, for observation of such radiation, the existence of two conditions is necessary (1) presence of cold matter and (2) presence of the electrons with energy sufficient for K-shell excitation, i.e., at least several $\mathrm{keV}$. As high energy electrons can be generated only after irradiation of the cluster by the main laser pulse, $\mathrm{K}_{\alpha}$ lines will be observed only in the case when the cold dense cluster core remains after irradiation by the laser prepulses.

As it is seen from Figure 9 (upper panel) at very high laser contrast $>10^{8}$ observable spectra contain intense $\mathrm{K} \alpha$ line which is not observed at contrast $2 \times 10^{5}$. It means that in the latter case the laser prepulse was strong enough to ionize all cluster atoms before the arrival of the main pulse. At higher laser contrasts the cold dense part of cluster survived after the laser prepulse. In such case, the Hollow ions and neutral $\mathrm{K}_{\alpha}$ spectral structures with wavelengths of about $4.18 \AA$ are clearly seen. Since generation of these states require hot electrons, generated by the main laser pulse, and cold dense plasma therefore an observation of such structure near $4.18 \AA$ will also suggest the existence of a dense core at the moment of arrival of the main pulse. Moreover, a comparison of the experimental and the calculated spectra of hollow ions can be used for estimation of the parameters of the most dense plasma regions generated by the laser prepulse. Such type of calculations were done by atomic and kinetic models (Colgan et al., 2011; Faenov et al., 2011) and their results show that the spectrum of the hollow ions obtained for laser contrast $10^{10}$, is described adequately by the modeling for the plasma with $T_{\mathrm{e}}=50 \mathrm{eV}$ and $N_{\mathrm{e}}=5 \times 10^{22} \mathrm{~cm}^{-3}$ in which there is $1 \%$ fraction of hot electrons.

\section{CONCLUSIONS}

During the past years many observations have been done on such exotic atomic objects as hollow ions. Their spectra have been obtained in the interaction of ultrashort high-power laser pulses with condensed targets (solid targets, clusters) and even with dense gases. So, the spectra of hollow ions are not exotic now. Their excitation is especially efficient in high-density plasmas and permits developing X-ray spectral methods for ultrahigh-density plasma diagnostics, particular, for diagnostic of warm dense matter and non-ideal plasmas. Indeed, hollow ions spectra are not sensitive to the non-ideality effects in plasma because they are emitted in the electron transitions between deep-lying inner shells of ions. By contrast, the applications of methods based on the spectral transitions between the outer shells of ions require a reconsideration of the existing collisional-radiative kinetic plasma models and, perhaps, for a new theory of the spectra of a strongly nonideal plasma.

With the development and employment of the plasma $\mathrm{X}$-ray lasers, $\mathrm{X}$-ray free-electron lasers and conventional visible lasers with ultra-high intensities, when Radiation Dominant Regime start to play a very important role, the spectra of hollow ions will gain more and more importance. In the experiments of such kind, the inner-shell ionization processes in atoms and ions, which are responsible for the production of hollow ions, are the principal mechanism of laser energy absorption, for any X-ray radiation source. In this case, produced plasma will be characterized by solid-state density and relatively low temperatures, i.e., will be a non-ideal plasma, with only few sufficient techniques appropriate for determining its parameters.

As it was mentioned above, the hollow ions must be also generated when plasma is heated by fast heavy ion beams. In this case, the resultant substance state will be also characterized by solid-state density, and some spatial regions of targets will have relatively low temperatures, i.e., will be also a non-ideal plasma. We consider that hollow ion spectra are promising diagnostic tool for such plasma too.

\section{ACKNOWLEDGMENTS}

This work was supported by the Russian Foundation for Basic Research under grant Nos \#14-22-02089 and 14-02-91171-GFEN_a.

\section{REFERENCES}

Abdallah, J. Jr., Skobelev, I.Yu., Faenov, A.Ya., Magunov, A.I., Pikuz, T.A., Flora, F., Bollanti, S., Di Lazzaro, P., Letardi, T., Burattini, E., Grilli, A., Reale, A., Palladino, L., Tomassetti, J., Scafati, A. \& Reale, L. (2000). Spectra of multiply charged hollow ions in the plasma produced by a short-wavelength nanosecond laser. Quant. Electron. 30, 694-702

Abdallah, J. Jr., Faenov, A.Ya., Skobelev, I.Yu., Magunov, A.I., Pikuz, T.A., Auguste, T., D’oliveira, P., Hulin, S. \& Monot, P. (2001). Hot electron influence on the X-ray emission spectra of Ar clusters heated by a high intensity 60 fs laser pulses. Phys. Rev. A 63, 032706.

Abdallah, J. JR., Csanak, G., Fukuda, Y., Akahane, Y., Aoyama, M., Inoue, N., Ueda, H., Yamakawa, K. Faenov, A.Ya., Magunov, A.I., Pikuz, T.A. \& Skobelev, I.Yu. (2003). Time dependent boltzmann-kinetic model of the X-rays produced by the ultrashort pulse laser irradiation of argon clusters. Phys. Rev. A 68, 063201 .

Aglitskiy, Y., Lehecka, T., Deniz, A., Hardgrove, J., Seely, J., Brown, C., Feldman, U., Pawley, C., Gerber, K., Bodner, S., Obenschain, S., Lehmberg, R., Mclean, E., Pronko, M., Sethian, J., Stamper, J., Schmitt, A., Sullivan, C., Holland, G. \& LAming, M. (1996). X ray emission from plasmas created by smoothed KrF laser irradiation. Phys. Plasmas 3, 3438-3447.

Ammosov, M.V., Delone, N.B. \& Krainov, V.P. (1986). Tunnel ionization of complex atoms and of atomic ions in an alternating electromagnetic field. Sov. Phys. JETP 64, 1191.

Andiel, U., Eidmann, K., Hakel, P., Mancini, R.C., Junkel-Vives, G.C., Abdallah, J. \& Witte, K. (2002). Demonstration of 
aluminum K-shell line shifts in isochorically heated targets driven by ultrashort laser pulses. Europhys. Lett. 60, 861.

Andiel, U., Eidmann, K., Witte, K., Uschmann, I. \& Förster, E. (2002a). Comparative study of time-resolved K-shell spectra from aluminum plasmas generated by ultrashort laser pulses at 395 and 790 nm. Appl. Phys. Lett. 80, 198.

Antici, P., Fuchs, J., D’humières, E., Lefebvre, E., Borghesi, M., Brambrink, E., Cecchetti, C.A., Gaillard, S., Romagnani, L., Sentoku, Y., Toncian, T., Willi, O., Audebert, P. \& Pépin, H. (2007). Energetic protons generated by ultrahigh contrast laser pulses interacting with ultrathin targets. Phys. Plasmas 14, 030701.

Atzeni, S. \& Meyer-Ter-Vehn, J. (2004). The Physics of Inertial Fusion. Oxford: Clarendon Press.

Auguste, T., Faenov, A.Ya., Fukumoto, I., Hulin, S., Magunov, A.I., Monot, P., D’oliveira, P., Pikuz, T.A., SAsaki, A., Sharkov, B.Yu., Skobelev, I.Yu., Tajima, T. \& Zhidkov, A.G. (2001). Observation of MeV multicharged ions and hot electrons accelerated by a 65-fs laser pulse J. Quant. Spectr. \& Rad. Trans. 71, 147-156.

Bailey, J., Carlson, A.L., Chandler, G., Derzon, M.S., Dukart, R.J., Hammel, B. A., Lockner, T. R., Maenchen, J., Mcguire, E.J., Mehlhorn, T.A., Nelson, W.E., Ruggles, L.E., Stygar, W.A. \& Wenger, D.F. (1990). Observation of Ko. X-ray satellites from a target heated by an intense ion beam. Lasers Part. Beams 8, 555.

Behar, E., Sako, M. \& Kahn, S.M. (2001). Soft X-ray absorption by $\mathrm{Fe}^{0+}$ to $\mathrm{Fe}^{15+}$ in active galactic nuclei. Astrophys. J. 563, 497-504.

Blasco, F., Stenz, C., Salin, F., Faenov, A.Ya., Magunov, A.I., Pikuz, T.A. \& Skobelev, I.Yu. (2001). Portable, tunable, highluminosity spherical crystal spectrometer with X-ray charge cuple device, for high-resolution X-ray spectromicroscopy of clusters, heated by femtosecond laser pulses. Rev. Sci. Instrum 72, 1956.

Boldarev, A.S., Gasilov, V.A. \& Faenov, A.YA. (2004). On the generation of large clusters in gas-jet targets for lasers. J. Tech. Phys. 49, 388.

Boldarev, A.S., Gasilov, V.A., Faenov, A.Ya., Fukuda, Y. \& YAMAKAWA, K. (2006). Gas-cluster targets for femtosecond laser interaction: Modeling and optimization. Rev. Sci. Instr. 77, 083112.

Briand, J.-P., Giardino, G., Borsoni, G., Essabaa, S., Briand, P., Geller, R., Desclaux, J.P., Bliman, S. \& Ristori, C. (1990). Production of hollow atoms by the excitation of highly charged ions in interaction with a metallic surface. Phys. Rev. Lett. 65, 159.

Briand, J.-P., Giardino, G., Borsoni, G., Froment, M., Eddrief, M., Sébenne, C., Bardin, S., Schneider, D., Jin, J., Khemliche, H., Xie, Z. \& Prior, M. (1996). Decay of hollow atoms above and below a surface. Phys. Rev. A. 54, 4136-4139.

Briand, J.-P., Schneider, D., Bardin, S., Khemliche, H., Jin, J., Xie, Z. \& Prior, M. (1997). X-ray studies of the interaction of N, O, and Ne hydrogen-like ions below surfaces. Phys. Rev. A. 55, 3947-3955.

Chabrier, G. (2009). Plasma physics and planetary astrophysics. Plasma Phys. Contr. Fusion 51, 124014.

Colgan, J., Abdallah, J. Jr., Faenov, A.Y., Pikuz, T.A., Skobelev, I.Yu., Fortov, V.E., Fukuda, Y., Akahane, Y., Aoyama, M., Inoue, N. \& Yamakawa, K. (2008). The role of hollow atoms in the spectra of an ultrashort-pulse-laser-driven Ar cluster target. Laser Part. Beams 26, 83-93.

Colgan, J., Abdallah, J. Jr., Faenov, A.Ya., Pikuz, T.A. \& SkobeLEV, I.YU. (2008a). MUTA calculations of laser-produced Mg hollow atom spectrum. Phys. Scripta 78, 015302.

Colgan, J., Abdallah JR., J. , Fontes, C.J., Kilcrease, D.P., Dunn, J., Purvis, M. \& LeE, R.W. (2010). Non-LTE and gradient effects in K-shell oxygen emission laser-produced plasma. Hi. Ener. Density Phys. 6, 295.

Colgan, J., Abdallah, J. JR., Faenov, A.Ya., Pikuz, T.A., Skobelev, I.Yu., Fukuda, Y., Hayashi, Y., Pirozhrov, A., Kawase, K., Shimomura, T., Kiriyama, H., Kato, Y., Bulanov, S.V. \& Kando, M. (2011). Observation and modeling of high resolution spectral features of the inner-shell X-ray emission produced by $10^{-10}$ contrast femtosecond-pulse laser irradiation of argon clusters. Hi. Ener. Density Phys. 7, 77.

Colgan, J., Abdallah, J. Jr., Faenov, A.Ya., Pikuz, S.A., Wagenatrs, E., Booth, N., Culfa, O., Dance, R.J., Evans, R.G., Gray, R.J., Kaempfer, T., Lancaster, P. Mckenna, A. L. RosSAll, I.Yu. Skobelev, K. S. Schulze, K.S., Uschmann, I., ZhiDKov, A.G. \& Woolsey, N.C. (2013). Exotic dense matter states pumped by relativistic laser plasma in the radiation dominant regime, Phys. Rev. Lett. 110, 125001.

DAIDO, H. (2002). Review of soft X-ray laser researches and developments. Rep. Prog. Phys. 65, 1513.

Diamant, R., Huotari, S., Hämäläinen, K., KaO, C.C. \& Deutsch, M. (2000b). Evolution from threshold of a hollow atom's X-ray emission spectrum: The $\mathrm{Cu} \mathrm{K}^{\mathrm{h}} \alpha_{1,2}$ Hypersatellites. Phys. Rev. Lett. 84, 3278.

Diamant, R., Huotari, S., Hämäläinen, K., KaO, C.C. \& Deutsch, M. (2000a). Cu $\boldsymbol{K}^{\boldsymbol{h}} \boldsymbol{\alpha}_{\mathbf{1}, \mathbf{2}}$ hypersatellites: Suprathreshold evolution of a hollow-atom X-ray spectrum. Phys. Rev. A. 62, 052519.

Diamant, R., Huotari, S., Hämäläinen, K., Sharon, R., Kao, C.C. \& Deutsch, M. (2001). Structure of the W $\mathbf{L} \boldsymbol{a}_{1,2}$ X-ray spectrum. Phys. Rev. A. 63, 022508.

Diamant, R., Huotari, S., Hämäläinen, K., Sharon, R., Kao, C.C. \& Deutsch, M. (2003). Diagram X-ray emission spectra of a hollow atom: The $\mathrm{K}^{\mathrm{h}} \alpha_{1,2}$ and $\mathrm{K}^{\mathrm{h}} \beta_{1,3}$ hypersatellites of $\mathrm{Fe}$. Phys. Rev. Lett. 91, 193001.

Elton, R.C. (1990). X-Ray Lasers. New York: Academic.

emma, P., Akre, R., Arthur, J., Bionta, R., Bostedt, C., Bozek, J., Brachmann, A., Bucksbaum, P., Coffee, R., Decker, F.-J., Ding, Y., Dowell, D., Edstrom, S., Fisher, A., Frisch, J., Gilevich, S., Hastings, J., Hays, G., Hering, P.H., Huang, Z., Iverson, R., Loos, H., Messerschmidt, M., Miahnahri, A., Moeller, S., Nuhn, H.-D., Pile, G., Ratner, D., Rzepiela, J., Schultz, D., Smith, T., Stefan, P., Tompkins, H., Turner, J., Welch, J., White, W., Wu, J., Yocky, G. \& Galayda, G. (2010). First lasing and operation of an angstrom-wavelength free-electron laser. Nat. Photon 4, 641-647.

Evans, R.G., Clark, E.L., Eagleton, R.T., Dunne, A.M., Edwards, R.D., Garbett, W.J., Goldsack, T.J., James, S., Smith, C.C., Thomas, B.R., Clarke, R., Neely, D.J. \& Rose, S.J. (2005). Rapid heating of solid density material by a petawatt laser. App. Phys. Lett. 86, 191505.

Faenov, A.Ya., Pikuz, S.A., Erko, A.I., Bryunetkin, B.A., Dyakin, V.M., Ivanenkov, G.V., Mingaleev, A.R., PikuZ, T.A., RomanoVA, V.M. \& SHELKovenKo, T.A. (1994). High-performance X-ray spectroscopic devices for plasma microsources investigations. Phys. Scripta 50, 333. 
Faenov, A.Ya., Abdallah, J., Clark, R.E.H., Cohen, J., Johnson, R.P., Kyrala, G.A., Magunov, A.I., Pikuz, T.A., Skobelev, I.Yu. \& WILKE, M.D. (1997). High-resolution X-ray spectroscopy of hollow atoms created in plasma heated by subpicosecond laser radiation. Proc. SPIE 3157, 10-21.

Faenov, A.Ya., Magunov, A.I., Pikuz, T.A., Skobelev, I.Yu., Pikuz, S.A., Urnov, A.M., Abdallah, J., Clark, R.E.H., Cohen, J., Johnson, R.P., Kyrala, G.A., Wilke, M.D., MaksimchuK, A., Umstadter, D., Nantel, N., Doron, R., Behar, E., Mandelbaum, P., Schwob, J.J., Dubau, J., Rosmej, F.B. \& Osterheld, A.L. (1999). High-resolved X-ray spectra of hollow atoms in a femtosecond laser-produced solid plasma. Phys. Scripta T80, 536.

Faenov, A.Ya., Pikuz, T.A., Skobelev, I.Yu., Fukuda, Y., Colgan, J. \& Abdallah, J. JR. (2009). Hollow ion spectra in warm dense laser-produced plasma: Observation and modeling. J. Phys. Conf. Ser. 163, 012016.

Faenov, A.Ya., Skobelev, I.Yu., Pikuz, T.A., Fortov, V.E., Boldarev, A.S., Gasilov, V.A., Chen, L.M., Zhang, L., Yan, W.C., YAun, D.W., MaO, J.Y. \& WANG, Z.H. (2011). Diagnostics of the early stage of the heating of clusters by a femtosecond laser pulse from the spectra of Hollow ions. JETP Lett. 94, 187-193.

Faenov, A.Ya., Skobelev, I.Yu., Pikuz, T.A., Pikuz, S.A. Jr., Fortov, V.E., Fukuda, Y., Hayashi, Y., PirozhKov, A., Kotaki, H., Shimomura, T., Kiriyama, H., Kanazawa, S., Kato, Y., Colgan, J., Abdallah, JR. J., Kando, M. \& J-KAREN Laser Operation Group. (2012). X-ray spectroscopy diagnoses of clusters surviving under prepulses of ultra-intense femtosecond laser pulse irradiation. Laser Part. Beams 30, 481-488.

ForTov, V.E. (2009). Extreme states of matter on Earth and in space. Phys. Uspekhi 52, 653.

Fournier, K.B., Faenov, A.Ya., Pikuz, T.A., Skobelev, I.Yu., BeLyaev, V.S., Vinogradov, V.I., Kyrilov, A.S., Matafonov, A.P., Bellucci, I., Martelluci, S.G., Petrocelli, S., Auguste, T., Hulin, S., Monot, P. \& D'oliveira, P. (2003). Influence of optical thickness and hot electrons on Rydberg spectra of Ne-like and F-like copper ions. Phys. Rev. E 67, 016402.

Gavrila, M. (1992). Atoms in Intense Laser Fields. New York: Academic Press, Inc.

Gauthier, J.-C., Geindre, J.-P., Audebert, P., Rousse, A., Dos Santos, A., Grillon, G., Antonetti, A. \& Mancini, R.C. (1995). Observation of $\mathrm{Kl}=\mathrm{LL}$ X-ray satellites of aluminum in femtosecond laser-produced plasmas. Phys. Rev. E 52, 2963.

Hansen, S., Shlyaptseva, A.S., Faenov, A.Y., Skobelev, I.Y., Magunov, A.I., Pikuz, T.A., Blasco, F., Dorchies, F., Stenz, C., Salin, F., Auguste, T., Dobosz, S., Monot, P., D’oliveira, P., Hulin, S., SAfronova, U.I. \& Fourneir, K.B. (2002). Hot electron influence on L-shell spectra of multicharged $\mathrm{Kr}$ ions generated in clusters irradiated by femtosecond laser pulses Phys. Rev. E, 66, 046412.

Hansen, S.B., Bauche, V., Bauche-Arnoult, C. \& Gu, M. F. (2007). Hybrid atomic models for spectroscopic plasma diagnostics. Hi. Ener. Density Phys. 3, 109.

Hansen, S.B., Colgan, J., Faenov, A.Ya., Abdallah, J. JR., Pikuz, S.A. Jr., Skobelev, I.Yu., WagenaArs, E., Booth, N., Culfa, O., Dance, R.J., Tallents, G.J., Evans, R.G., Gray, R.J., KaEmPfer, T. , Lancaster, K.L., Mckenna, P., Rossall, A.K., Schulze, K.S., Uschmann, I., Zhidkov, A.G. \& Woolsey, N.C. (2014). Detailed analysis of hollow ions spectra from dense matter pumped by X-ray emission of relativistic laser plasma. Phys. Plasmas 21, 031213.

Kalman, T.R. (2010). Modeling of photoionized plasmas. Space Sci. Rev. 157, 177-191.

Kawamura, T., Horioka, K. \& Koike, F. (2011). Ko radiation from low charge chlorine heated by an ion beam for cold dense plasma diagnostics. Laser Part. Beams 29, 135-140.

LANDAU, L. \& LifshitZ, E. (1961). The Classical Theory of Fields. London: Pergamon Press.

Limburg, J., Schippers, S., Hoekstra, R., Morgenstern, R., Kurz, H., Aumayr, F. \& Winter, H.P. (1995). Do hollow atoms exist in front of an insulating $\mathrm{LiF}(100)$ surface? Phys. Rev. Lett. $\mathbf{7 5}$, 217-221.

Macfarlane, J.J., Wang, P., Bailey, J., Mehlhorn, T.A., Dukart, R.J. \& Mancini, R.C. (1993). Analysis of $\mathrm{K}_{\alpha}$ line emission from aluminum plasmas created by intense proton beams. Phys. Rev. E 4, 2748.

Maksimchuk, A., Nantel, M., Ma, G., Gu, S., Cote, C.Y., UMstadter, D., Pikuz, S.A., Skobelev, I.Yu. \& Faenov, A.Ya. (2000). X-Ray radiation from matter in extreme conditions. JQSRT 65, 367.

Mazevet, S. \& Abdallah, J. JR. (2006). Mixed UTA and detailed line treatment for mid- $Z$ opacity and spectral calculations. J. Phys. B 39, 3419.

Mcmahon, S.J., Kavanagh, A.P., Watanabe, H., Sun, J., Tona, M., NaKamura, N., Ohtani, S. \& Currell, F.J. (2011). Characterization and parametrization in terms of atomic number of $\mathrm{X}$-ray emission from K-shell filling during ion-surface interactions. Phys. Rev. A. 83, 022901.

Mimura, H., Yumoto, H., Matsuyama, S., Koyama, T., Tono, K., Inubushi, Y., Togashi, T., Sato, T., Kim, J., Fukui, R., SAno, Y., Yabashi, M., Ohashi, H., IshiKawa, T. \& Yamauchi, K. (2014). Generation of $10^{20} \mathrm{Wcm}^{2}$ hard X-ray laser pulses with two-stage reflective focusing system. Nat. Commun. 5, 3539.

McPherson, A., Thompson, B.D., Borisov, A.B., Boyer, K. \& RHodES, C.K. (1994). Multiphoton-induced X-ray emission at 4-5 keV from Xe atoms with multiple core vacancies. Nat. 370, 631 .

Moribayashi, K., Sasaki, A. \& Zhidkov, A. (2001). Productions of hollow atoms from solids irradiated by high intensity laser. Phys. Scripta T92, 185.

Moribayashi, K., Suto, K., Zhidkov, A., Sasaki, A. \& Kagawa, T. (2001a). X-ray emission from hollow atoms produced by collisions of multiply charged ions with a solid. Laser Part. Beams 19, 643.

Nakamura, T., Koga, J.K., Esirkepov, T.Zh., Kando, M., Korn, G. \& Bulanov, S.V. (2012). High-power $\gamma$-ray flash generation in ultraintense laser-plasma interactions. Phys. Rev Lett. 108 , 195001.

Nakano, Y., Suda, S., Hatakeyama, A., Nakai, Y., Komaki, K., Takada, E., Murakami, T. \& Azuma, T. (2012). Selective production of the doubly excited $2 p^{2}\left({ }^{1} D\right)$ state in He-like $\mathrm{Ar}^{16+}$ ions by resonant coherent excitation. Phys. Rev. A $\mathbf{8 5}$, 020701(R).

Ninomiya, S., Yamazaki, Y., Koike, F., Masuda, H., Azuma, T., Komaki, K., Kuroki, K. \& SeKiguchi, M. (1997). Stabilized hollow ions extracted in vacuum. Phys. Rev. Lett. 78, 4557-4561

Nozaw, S., Iтон, N. \& Конуама, Y. (1998). Relativistic thermal bremsstrahlung gaunt factor for the intracluster plasma. Astrop. J. 507, 530 . 
Pandit, R.R. \& Sentoku, Y. (2012). Higher order terms of radiative damping in extreme intense laser-matter interaction, Phys. Plasmas 19, 073304.

Pikuz, S.A. Jr., Efremov, V.P., Rosmej, O., Blazevic, A., Korostiy, S., Fertman, A., Shutov, A.V., Norman, G.E. \& Hoffmann, D.H.H. (2006). Investigations of heavy-ion tracks' energy deposition inside solid media by methods of X-ray spectroscopy. J. Phys. A 39, 4765.

Pikuz, S.A. Jr., Faenov, A.Ya., Colgan, J., Dance, R.J., Abdallah, J., Wagenaars, E., Booth, N., Culfa, O., Evans, R.G., Gray, R.J., Kaempfer, T., Lancaster, K.L., Mckenna, P., Rossall, A.L., Skobelev, I.Yu., Schulze, K.S., Uschmann, I., Zhidkov, A.G. \& Woolsey, N.C. (2013). Measurement and simulations of hollow atom X-ray spectra of solid-density relativistic plasma created by high-contrast PW optical laser pulses. Hi. Ener. Density Phys. 9, 560.

Ridgers, C.P., Brady, C.S., Duclous, R., Kirk, J.G., Bennett, K., Arber, T.D., Robinson, A.P.L. \& Bell, A.R. (2012). Dense electron-positron plasmas and ultraintense $\gamma$ rays from laserirradiated solids. Phys. Rev. Lett. 108, 165006.

RoccA, J.J. (1999). Table-top soft X-ray lasers. Rev. Sci. Instrum. 70, 3799.

Rosmej, F.B., Faenov, A.Ya., Pikuz, T.A., Magunov, A.I., Skobelev, I.Iu., Auguste, T., D'oliveira, P., Hulin, S., Monot, P., Andreev, N.E., Chegotov, M.V. \& Veisman, M.E. (1999). Charge exchange induced formation of hollow atoms in high intensity laser-produced plasmas. J. Phys. B 32, L107.

Rosmes, F.B., Funk, U.N., Geissel, M., Hofmann, D.H.H., Tausehwitz, A., Faenov, A.Ya., Pikuz, T.A., Skobelev, I.Yu., Flora, F., Bollanti, S., Di Lazzaro, P., Letardi, T., Grilli, A., Palladino, L., Reale, A., Scafati, A., Reale, L., Auguste, T., D’oliveira, P., Hulin, S., Monot, P., Maksimchuk, A., Pikuz, S.A., Umstadter, D., Nanatel, M., Bock, R., Dornik, M., Stetter, M., Stoewe, S., YAKushev, V., Kulish, M. \& SHILKIN. (2000). X-Ray radiation from ions with K-shell vacanciesl. JQSRT 65, 477.

Rosmej, F.B., More, R., Rosmej, O.N., Wieser, J., Borisenko, N., Shevelko, V.P., Geißel, M., Blazevic, A., Jacoby, J., Dewald, E., Roth, M., Brambrink, E., Weyrich, K., Hoffmann, D.H.H., GoluBev, A.A., Turtikov, V., Fertman, A., Sharkov, B.Yu., Faenov, A.YA., Pikuz, T.A., Magunov, A.I. \& Skobelev, I.Yu. (2002). Methods of charge-state analysis of fast ions inside matter based on their X-ray spectral distribution Laser Part. Beams 20, 479.

Rosmej, O.N., Pikuz, S.A. JR., Wieser, J., Blazevic, A., Brambrink, E., Roth, M., Efremov, V.P., Faenov, A.YA., Pikuz, T.A., SkobeLEV, I.Yu. \& Hoffmann, D.H.H. (2003). Investigation of the projectile ion velocity inside the interaction media by the X-ray spectromicroscopy method. Rev. Sci. Instrum. 74, 5039.

Rosmej, O.N., Blazevic, A., Korostiy, S., Bock, R., Hoffmann, D.H.H., Pikuz, S.A. Jr., Efremov, V.P., Fortov, V.E., Fertman, A., Mutin, T., Pikuz, T.A. \& Faenov, A.YA. (2005b). Charge state and stopping dynamics of fast heavy ions in dense matter. Phys. Rev. A 72, 052901

Rosmej, O.N., Pikuz, S.A. Jr., Korostiy, S., Blazevic, A., Brambrink, E., Fertman, A., Mutin, T., Efremov, V.P., Pikuz, T.A.,
Faenov, A.Ya., Loboda, P., Golubev, A.A. \& Hoffmann, D.H.H. (2005a). Radiation dynamics of fast heavy ions interacting with matter. Laser Part. Beams 23, 1.

Rzadkiewicz, J., Gojska, A., Rosmej, O., Polasik, M. \& SŁabkowSKA, K. (2010). Interpretation of the Si $\boldsymbol{K}_{\boldsymbol{\alpha}}$ X-ray spectra accompanying the stopping of swift $\mathrm{Ca}$ ions in low-density $\mathrm{SiO}_{2}$ aerogel. Phys. Rev. A 82, 012703.

SARACHIK, E.S. \& Schappert, G.T. (1970). Classical theory of the scattering of intense laser radiation by free electrons. Phys. Rev. D 1, 2738.

Schenkel, T., Hamza, A.V., Barnes, A.V. \& Schneider, D.H. (1999). Interaction of slow, very highly charged ions with surfaces. Prog. Surf. Sci. 61, 23.

Skobelev, I.Yu., Faenov, A.Ya., Bryunetkin, B.A., Dyakin, V.M., Pikuz, T.A., Pikuz, S.A., Shelkovenko, T.A., Romanova, V.M. \& MingaleEv, A.R. (1995). Investigating the emission properties of plasma structures with X-ray imaging spectroscopy methods. J. Exper. Theor. Phys. 81, 692.

Skobelev, I.Yu., Faenov, A.Ya., Pikuz, T.A. \& Fortov, V.E. (2012). Hollow ions spectra in high density laser plasma. Phys. Uspekhi 182, 9.

Suckewer, S. \& JAEGLE, P. (2009). X-ray lasers: Past, present, future. Laser Phys. Lett. 6, 411.

Urnov, A.M., Dyubo, J., Faenov, A.Ya., Pikuz, T.A., Skobelev, I.Yu., Abdallah, J., Clark, R.E.H., Cohen, R.E.H., Johnson, R.P., Kyrala, G.A., Wilke, M.D. \& Osterheld, A.L. (1998). $\mathrm{X}$-ray spectra of multicharged hollow ions emitted by femtosecond laser plasma. JETP Lett. 67, 489-494.

Vinko, S.M., Ciricosta, O., Cho, B.I., Engelhorn, K., Chung, H.-K., Brown, C.R.D., Burian, T., Chalupský, J., Falcone, R.W., Graves, C., Hásková, V., Higginbotham, A., Juha, L., Krzywinski, J., Lee, H. J., Messerschmidt, M., Murphy, C.D., Ping, Y., Scherz, A., Schlotter, W., Toleikis, S., Turner, J.J., Vysin, L., Wang, T., Wu, B., Zastrau, U., Zhu, D., Lee, R.W.P., Heimann, A., NAgler, B. \& Wark, J.S. (2012). Creation and diagnosis of a solid-density plasma with an X-ray freeelectron laser. Nat. 482, 59-62.

Wang, P., MacFarlane, J.J. \& Moses, G.A. (1993). Relativisticconfiguration-interaction calculations of $K_{\alpha}$ satellite properties for aluminum plasmas created by intense proton beams Phys. Rev. E 48, 3934.

Winter, H. \& Aumay, F. (1999). Hollow atoms. J. Phys. B 32, R39.

ZhIDKOV, A. \& SASAKI, A. (2000). Effect of field ionization on interaction of an intense subpicosecond laser pulse with foils. Phys. Plasmas 7, 1341.

Zhidkov, A.G., Sasaki, A., Fukumoto, I., Tajima, T., Auguste, T., D’olivera, P., Hulin, S., Monot, P., Faenov, A.Ya., Pikuz, T.A. \& SkobeLEV, I.Yu. (2001). Transient distribution of energetic particles produced by intense femtosecond laser pulses irradiating solids. Phys. Plasma $\mathbf{8}, 3718$.

Zhidkov, A., Koga, J., Sasaki, A. \& Uesaka, M. (2002). Radiation Damping Effects on the Interaction of Ultraintense Laser Pulses with an Overdense Pla8, 185002 (2002) 